\author{
Military Technical College \\ Kobry El-Kobbah, \\ Cairo, Egypt
}

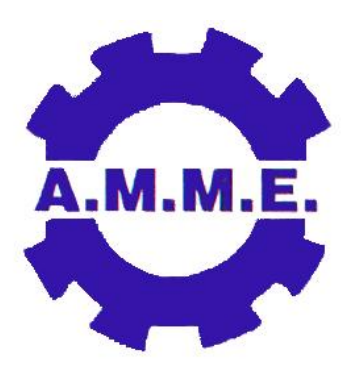

14th International Conference on Applied Mechanics and Mechanical Engineering.

\title{
Effect of Orthopaedic Stem Design on Femur Stress_Shielding
}

By
M. E. Abo-Elnor *
M. S. Abo_Elkhair*
A. E. Radi **

\begin{abstract}
:
Total joint replacement has become a widely accepted treatment for many destructive joint diseases including osteoarthritis and severe pathologic fractures. Of total joint replacements, the hip is one of the most commonly replaced joints. Hip stem design varies from long to short, thick to thin and matt to smooth. In this study the effect of hip stem length on bone remodeling after surgery which knows as stress-shielding effect was analyzed using a finite element technique. Results show that stress-shielding introduced when using long-stem hip is extended that that of using short-stem hip in a total hip replacement.
\end{abstract}

\section{Keywords:}

Femur stress-shielding, Biomechanics, Femur modeling, cemented hip implant 


$\begin{array}{ll}* * & \text { Egyptian Armed Forces } \\ * * & \text { Professor of orthopedics, Faculty of medicine Ain-Shams Univ. Cairo, Egypt }\end{array}$

\section{Introduction:}

The total hip replacement is widely employed to treat bone disease or in fracture repair. Although this is a successful treatment, some problems associated with the prosthesis design remain unsolved. One of these problems is the loss of bone on the proximal femur due to the effect of stress-shielding [1], [2]. It can lead to fracture, loosening and reduction in the amount of bone available for a revision surgery
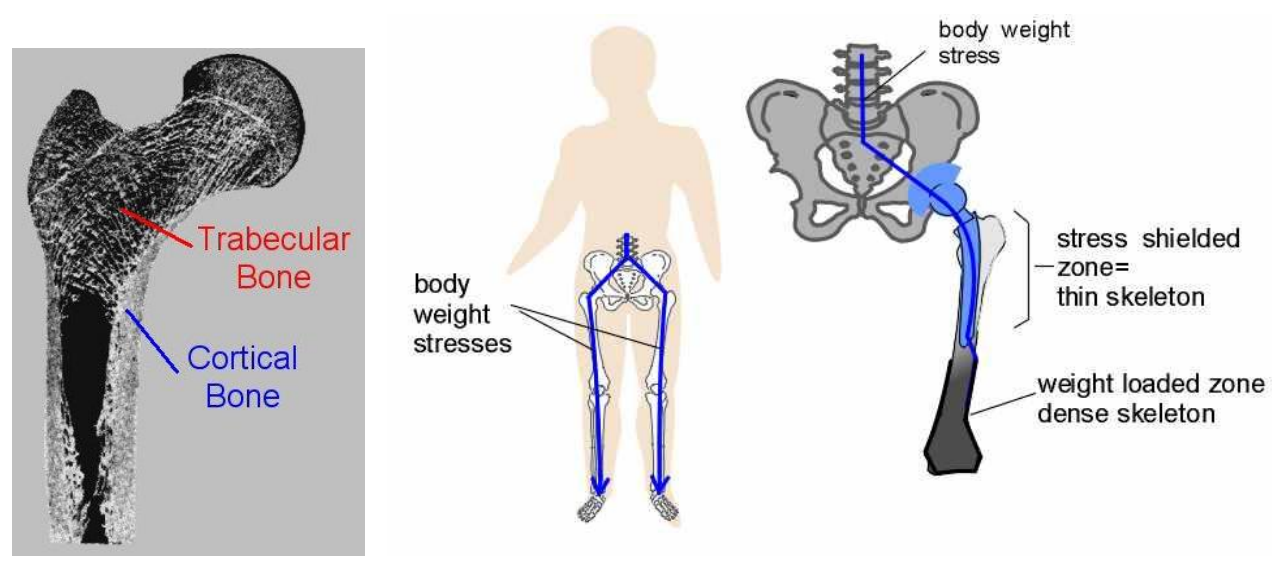

Fig. 1, Femur stress-shielding zone

The loosening mechanism is induced mainly by stress shielding which initiates the bone remodeling [3]. This makes the femur change its osseous density and distribution and therefore causes loosening of the joint stem. Stress shielding zone (Fig. 1) occurs due to the fact that stress is essentially carried by the stem, and consequently the stress on bone decreases relative to the intact femur (without prosthesis). The loss of bone is obviously related with the prosthesis design: a stiffer stem leads to a large amount of bone loss. This stiffness depends on stem dimensions as well as on stem material [4]. The dependence of the bone resorption on mechanical loads was described a century ago by Wolff's Law [5]. According to this law, the trabecular bone is able to self-adapt depending on the mechanical environment. In this work a numerical model using the finite element technique is carried out to analysis the effect of hip stem length on the level of stressshielding.

\section{Materials and Methods}

ANSYS finite element method software is used to establish an analysis model and simulate stress distribution of the femur bone when a cemented stem is used. Inventor, one of the advanced CAD modeling software, is used to generate two proposal models for a total hip with long stem and short stem to be used in the analysis of the effect of stem geometry on stress-shielding zone (Fig. 2). Advanced computed tomography (CT) data is used to create 3D CAD model of the femur bone.

Adaptive mesh technique is used to generate finite element mesh of the hip joint stem, bone and bone cement in the software (Fig. 3). Stem material of hip joint is that of Ti-6Al- 
4V with Young's modulus $110000 \mathrm{MPa}$ and Poisson's ratio 0.3. Material of the bone cement used is Ultrahigh- molecular-weight (UHMW) polyethylene with Young's modulus of $2280 \mathrm{MPa}$ and Poisson's ratio 0.3.
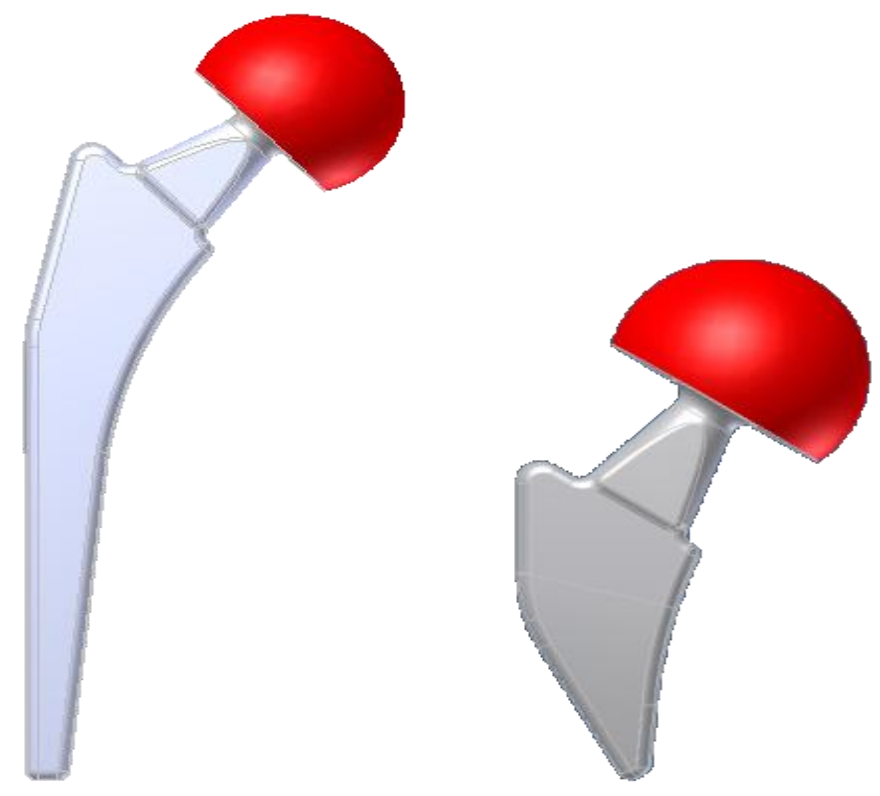

Fig. 2, Long-stem (left) and Short-stem total hip replacement implant

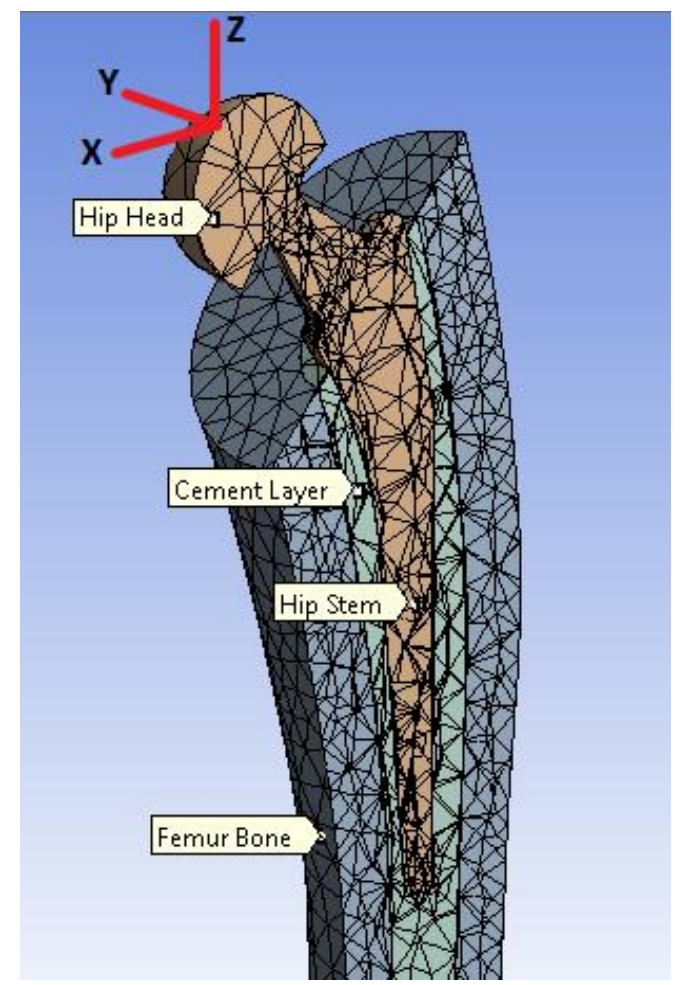

Fig. 3, Section view in the meshed model with cooridinate system

The bone within the femur has a Young's modulus of $1300 \mathrm{MPa}$ and Poisson's ratio 0.28 [6]. It should be noted, however, that all materials were assumed to be isotropic in the present study. In reality both the fiber-reinforced epoxy of the composite femur and the cancellous and cortical bone are known to be anisotropic. The Patient weight is supposed 
to be 85 kilograms in the analysis, wouldn't consider its initial conditions, such as sex, age, activity, etc. The load state in gait cycle of human body is equivalent to 4.6 times of body weight (BW), at 45 present of the gait cycle, to act on femur head. The load resolves into $F x=-1500 \mathrm{~N}, F y=-600 \mathrm{~N}, F z=-3500 \mathrm{~N}$ with $\mathrm{x}, \mathrm{y}, \mathrm{z}$ (Fig. 3) and applied as a quasi-static.

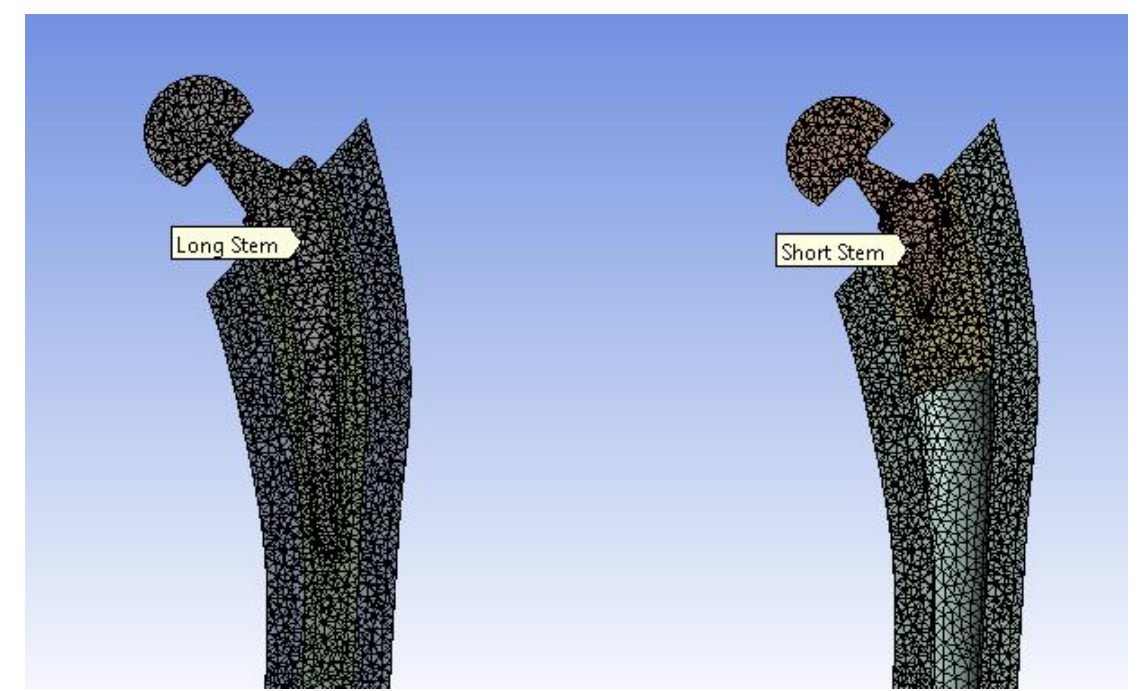

Fig. 4, Rrfined mesh for long-stem hip (left) and short-stem hip (right)

For comparative analysis, the following parameters were considered and compared; stress in the cement layer, and stress in the bone around the cement layer. Completely bonded contact type was chosen as contact condition for contact surfaces. A total hip replacement case was studied using a long hip stem and a short one in the same analysis platform as shown in Fig.4, a refined mesh is used and the bottom boundary of the femur is set to be fixed resembles the knee connection.

\section{Results and Discussion}

The analysis was carried out firstly by omitting the torsion component of the applied force (i.e. Fy=0). Fig. $(5,6)$ show the equivalent stress distribution from the hip to the femur bone through the cement layer. The low stress zone of the long-stem hip is extended than that of the short-stem hip and hence the stress-shield zone of the long-stem hip is greater than that of the short-stem hip. The conclusion can be drawn from Fig. (7), which resemble the elastic strain distribution over the analyzed model, the elastic strain zone of the long-stem hip is greater than the one of short-stem hip which means low strain distribution over the femur top when using the long-stem hip and hence weak bone remolding in this zone after surgery. 


\section{B: Comparizon \\ Equivalent Stress \\ Unit: $\mathrm{Pa}$ \\ Time: 1 \\ 22-Dec-09 12:21 AM \\ 2.7534e8 Max \\ $1.4697 \mathrm{e} 8$ \\ $7.8452 \mathrm{e} 7$ \\ $4.1877 \mathrm{e} 7$ \\ $2.2354 \mathrm{e} ?$ \\ $1.1932 \mathrm{e} 7$ \\ $6.3693 \mathrm{e} 6$ \\ $3.3999 \mathrm{e} 6$ \\ $1.8148 \mathrm{e} 6$ \\ $9.6874 \mathrm{e} 5$ \\ $5.1711 \mathrm{e} 5$ \\ $2.7603 \mathrm{e} 5$ \\ $1.4734 \mathrm{e} 5$ \\ 78649 \\ 41982 Min}

Type: Equivalent (von-Mises) Stress
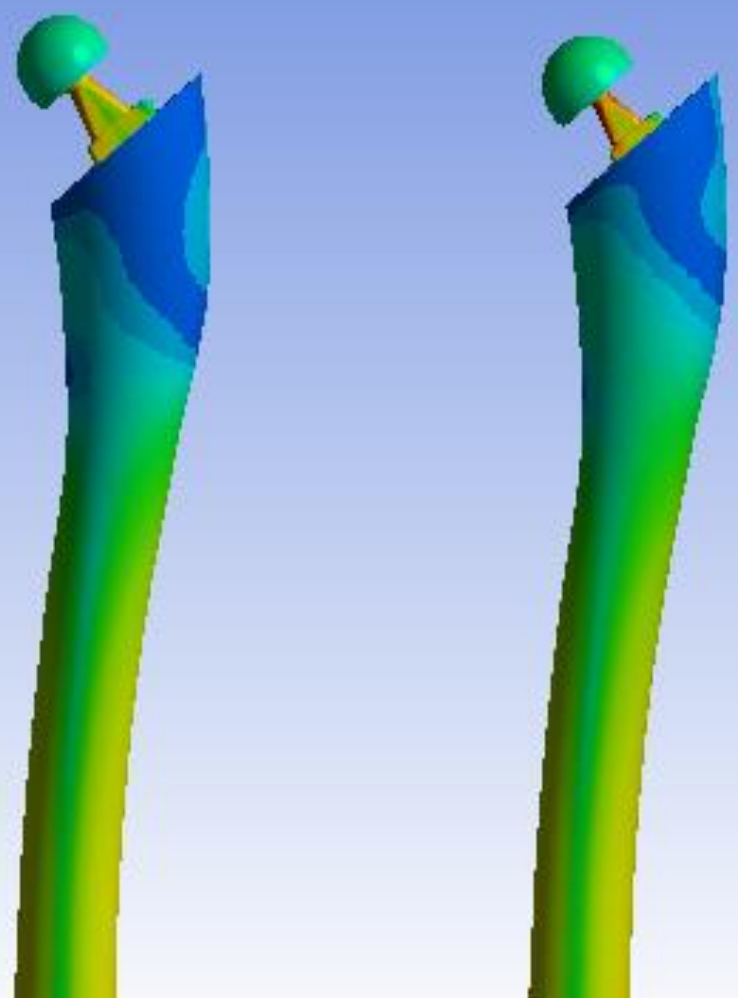

Fig. 5, Stress distribution on long-stem hip (left) and short-stem hip (right)

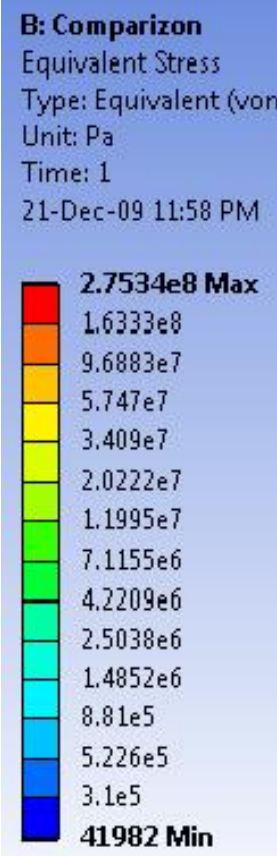

Fig. 6, Stress distribution on long-stem hip (left) and short-stem hip (right) in section view 


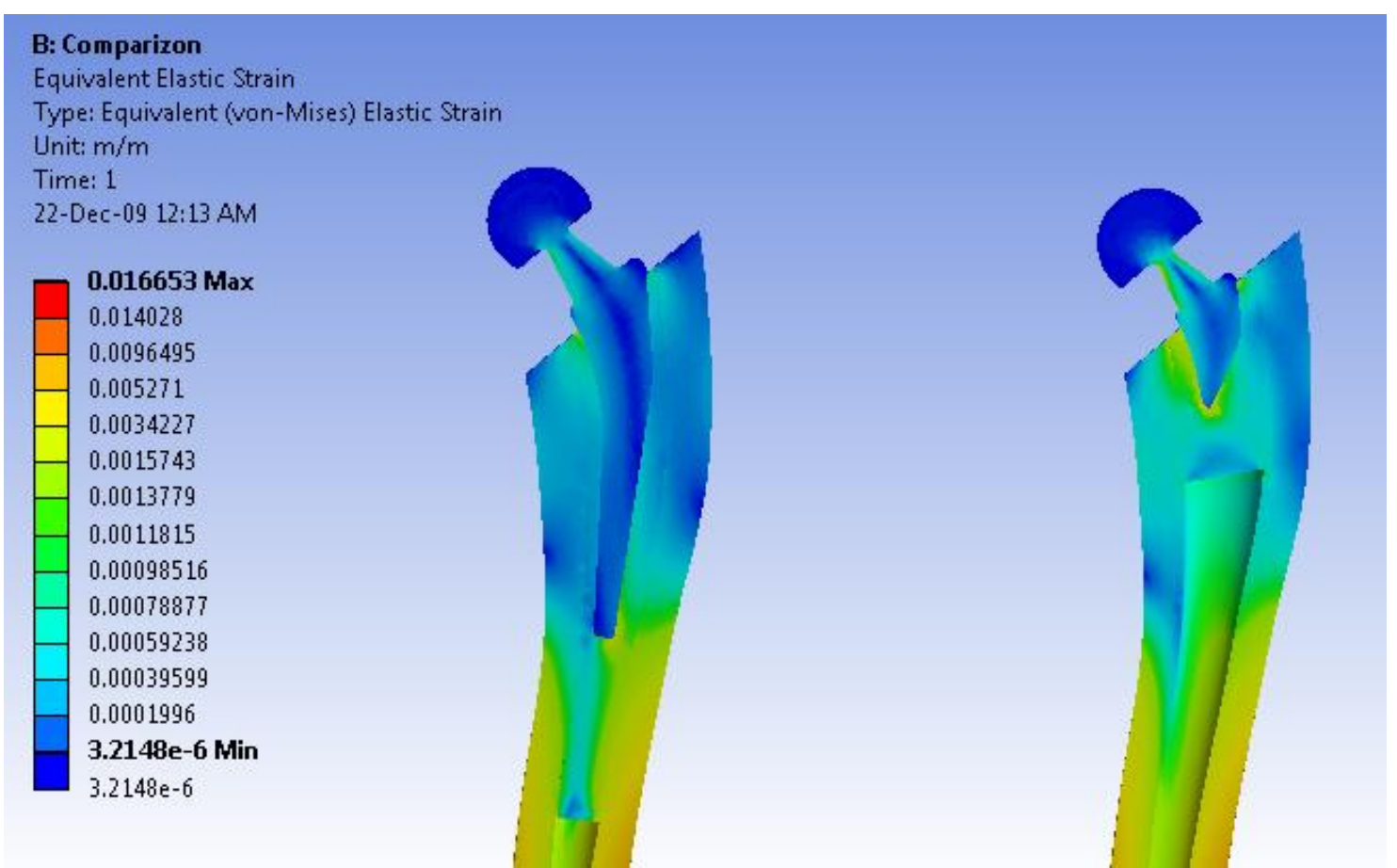

Fig. 7, Elastic strain on long-stem hip (left) and short-stem hip (right) in section view

The stress distribution over $360^{\circ}$ is obtained by lateral cross sections of the modeled femur and plotted in Fig. (8), the figure shows the extended distribution of the stress-shield zone of the long-stem hip over the one of short-stem hip.
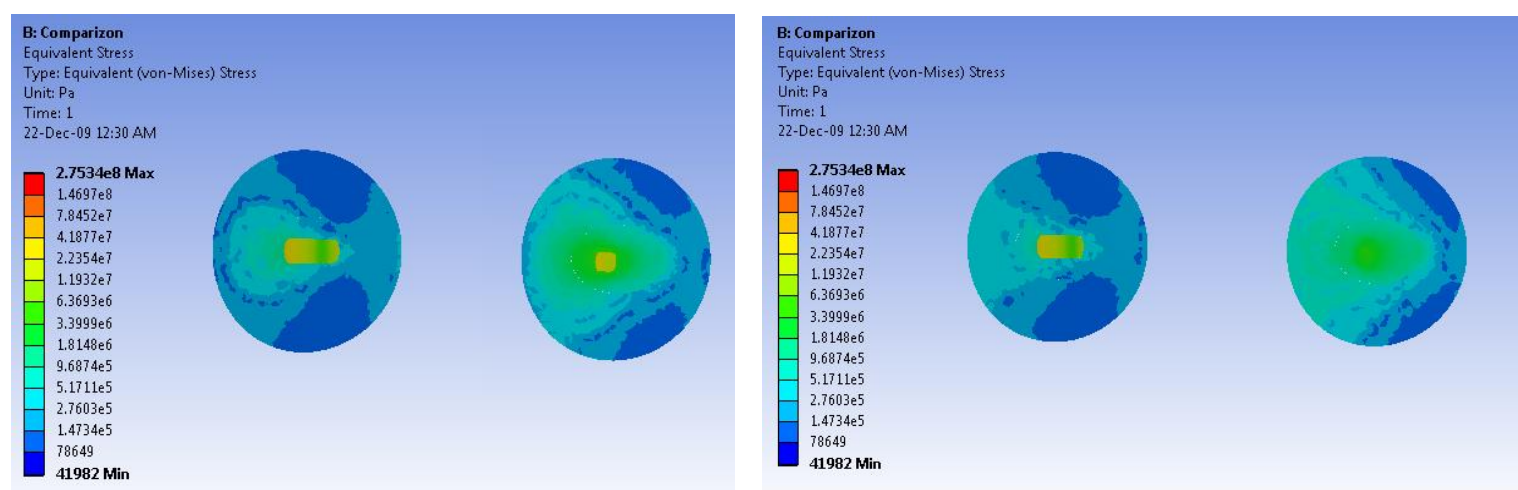

Fig. 8, Stress distribution on long-stem hip (left) and short-stem hip (right) in consequetive lateral cross sections of the femur; $4 \mathrm{~cm}$ from top (left), $8 \mathrm{~cm}$ from top (right)

When a tensional component of the applied force is activated in the analysis as a result of patient stairs climb or ramp walking, the stress-shield zone rotate around the femur vertical axes as a result introducing overload to the normally unloaded zone which may leads to femur cracks. In fact this may be a hot topic of discussion. The fixation advantage of the long-stem hip regardless its extended stress-shielding makes long-stem hip preferable to the surgeon while short-stem hip over comes the extended stress-shielding requires professional surgeon. 

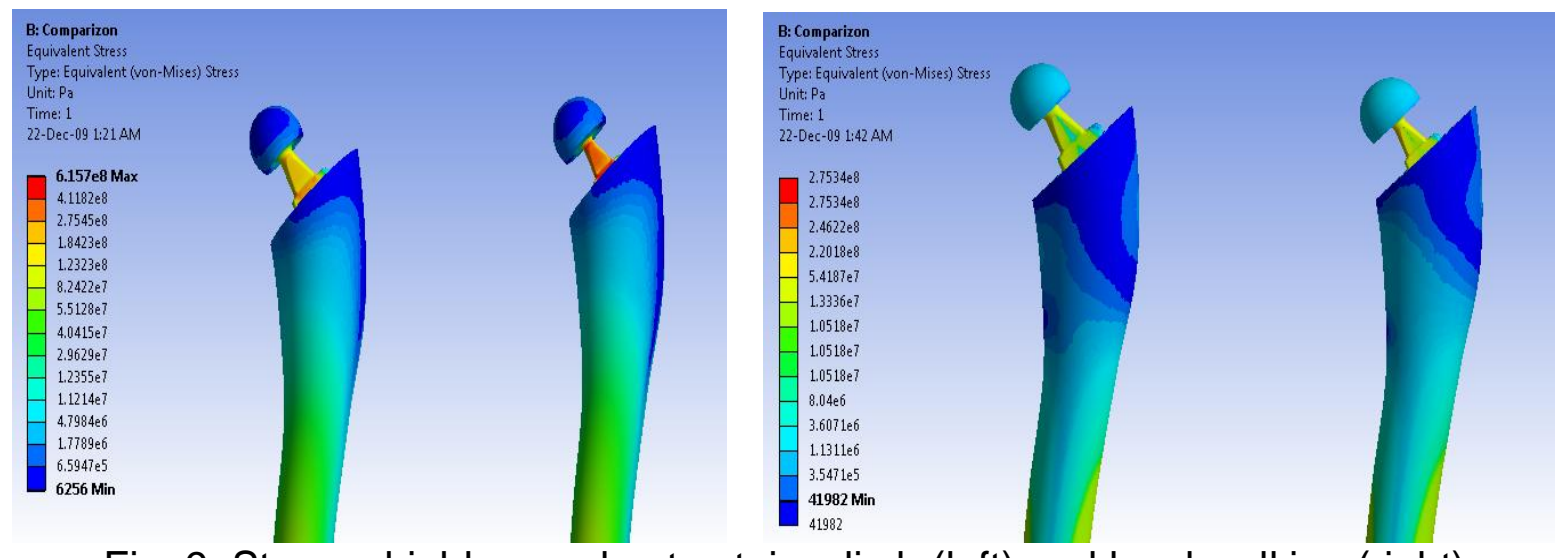

Fig. 9, Stress-shield zone due to stairs climb (left) and level walking (right)

\section{Conclusions}

The finite element analysis conducted in this work reveals the effect of stem geometry (length) on the creation of what so named stress-shielding of the femur bone after surgery. Regardless the ease of implant of a long-stem hip; the analysis shows that using shortstem hip, in total hip replacement, is better than using long-stem hip from the stressshielding point of view. Applying torsion stress to the femur after surgery as a result of patient practice may decrease the possibility of stress-shield zone extension over the femur bone and hence allow condense bone remodeling around the implant and long-time strengthen the bone after surgery. As a conclusion, using of long-stem hip is suitable for younger patients who can do heavy practice after surgery to minimize stress-shielding while short-stem hip is much more suitable for elders.

\section{References:}

[1] J. D. Bobyn, A. H. Glassman, et al , "The Effect of Stem stiffness on Femoral Bone Resorption after Canine Porous-Coated Total Hip Arthroplasty", Clinical Orthopaedics and Related Research Vol. 261, PP. 196-213, 1990

[2] B. Rietbergen, R. Huiskes, et al, "The Mechanism of Bone Remodeling and Resorption around Press-Fitted THA Stems", J. Biomechanics Vol. 26, No 4/5, PP. 369-382, 1993

[3] CHEUNG-HWA HSU, "TRADE-OFF DESIGN OF TOTAL HIP PROSTHESIS", Biomed Eng Appl Basis Comm Vol. 17, PP. 319-323, 2005

[4] H. Weinans, R. Huiskes and H. J. Grootenboer, "Effects of Material Properties of Femoral Hip Components on Bone Remodeling", J. Orthop. Res. Vol. 10, No 6, PP. 845-853, 1992

[5] J. Wolff, "The law of Bone Remodelling", Translated by Maquet P. and Furlong R. Springer, Berlin, (1986).

[6] Fagan, M. J., and Lee, A. J. C., "Materials Selection in the Design of the Femoral Component of Cemented Total Hip Replacement", Clin. Mater. , PP.151-167, 1986. 